\section{In vivo confocal \\ microscopy of congenital aniridia-associated keratopathy}

\begin{abstract}
Purpose To explore the in vivo morphological changes of cornea and limbus in aniridia-associated keratopathy (AAK). Methods Three cases of AAK were examined with the application of in vivo confocal microscopy (IVCM).

Results Abnormal structure of wing and basal layer of epithelium, the loss of subbasal nerves, and the presence of goblet cells at central cornea could be identified in the most severe case, along with the absence of Vogt palisades. The less extent of abnormalities in corneal epithelial cells, subbasal nerve, and Vogt palisades were visible in the moderate or mild cases. Conclusions The morphological changes of cornea and limbus vary in AAK, and IVCM is a promising tool to determine the degree of limbal stem cell deficiency in patients with AAK.

Eye (2013) 27, 763-766; doi:10.1038/eye.2013.50; published online 12 April 2013
\end{abstract}

Keywords: aniridia-associated keratopathy; in vivo confocal microscopy; cornea; limbus

\section{Introduction}

Limbal stem cell deficiency (LSCD) is often involved in congenital aniridia. ${ }^{1}$ Little is known regarding the in vivo morphological characteristics of the cornea and limbus in aniridia-associated keratopathy (AAK). These microstructural changes have great significance for the classification of the disease and the selection of appropriate treatment. Therefore, we investigate the morphological characteristics
Q Le', SX Deng ${ }^{2}$ and $\mathrm{J} X \mathrm{u}^{1}$

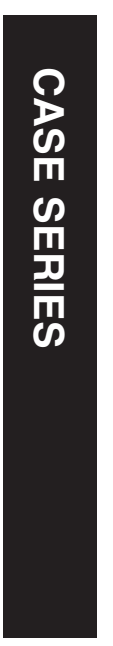

of cornea and limbus in three cases of congenital aniridia using in vivo confocal microscopy (IVCM) (HRTII/RCM).

\section{Case reports}

Case 1

A 58-year-old Chinese woman complained of constant red eye, photophobia, and unsatisfactory vision recovery after phacoemulsification and intraocular lens implantation, which were performed 3 months earlier. The invasion of vascular pannus into peripheral half of the cornea, as well as subepithelial and stromal opacity was identified under the slitlamp biomicroscopy (Figure 1a). IVCM showed complete loss of normal wing and basal epithelial cells, the presence of goblet cells within corneal epithelium, subepithelial fibrosis, and the absence of subbasal nerves at central cornea, and the entire loss of palisades of Vogt at each quadrant of the limbus (Figures 1b-f).

\section{Case 2}

The invasion of $2-3 \mathrm{~mm}$ vascular pannus into the cornea and focal subepithelial fibrosis at the central cornea was identified in a 54-year-old Chinese woman (Figure 2a). IVCM on the central cornea showed abnormal structure of the wing and basal layer of epithelium, subepithelial fibrosis, infiltration of dendritic cells, and the absence of subbasal nerves. Significantly decreased cellular density of wing epithelium, basal epithelium, and stroma was found in this patient, compared with age- and gender-matched control (Table 1). At each quadrant of the limbus, the palisades of Vogt were absent (Figures 2b-f).
1Department of Ophthalmology, Eye \& ENT Hospital of Fudan University, Shanghai, China

${ }^{2}$ Department of Cornea and Uveitis, The Jules Stein Eye Institute, David Geffen School of Medicine, University of California, Los Angeles, CA, USA

Correspondence: J Xu,Department of Ophthalmology, Eye \& ENT Hospital of Fudan University, No. 83 Fenyang Road, Shanghai 200031, China.

Tel: +86 2164377134 Fax: +86216437 7151

Email: jianjiang-xu@ 163.com

Received: 22 October 2012 Accepted in revised form: 16 February 2013 Published online: 12 April 2013 

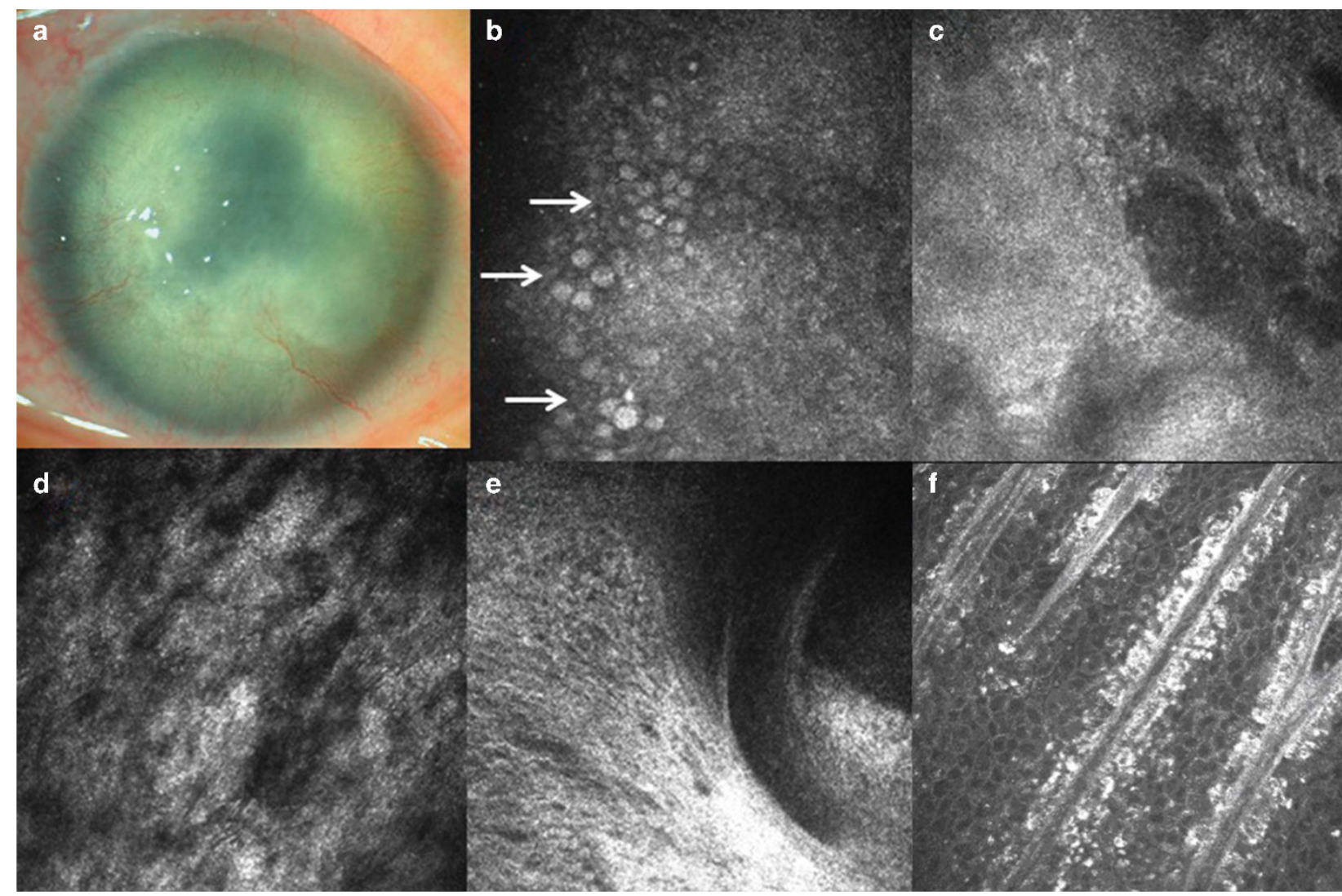

Figure 1 Clinical findings in case 1. (a) Slitlamp microscopic photo. (b-d) Confocal images of the cornea. Many goblet cells are present in the cornea (white arrows) (b). There is the absence of subbasal nerves (c). Stromal keratocytes are indistinguishable (d). (e-f) Confocal images of the limbus. The entire absence of Vogt palisades was found at limbus of this patient (e). In contrast, typical morphology of Vogt palisades was visible in the age-matched healthy subject (f).

\section{Case 3}

This 26-year-old man is the son of the patient in case 2. He presented with slight nystagmus, mild conjunctival hyperemia, relatively clear cornea, and posterior capsular cataract (Figure 3a). IVCM revealed almost normal morphology of corneal wing and basal epithelial cells except the prominent nuclei in the basal cells. Although subbasal nerve plexus were visible, the nerve density decreased compared with age-matched healthy subject, as shown inTable 1. The palisade-like stromal cords, rather than typical Vogt palisades, were found at the limbal area. However, the normally present hyperreflective cells around the stromal cords were absent (Figures 3b-h).

\section{Discussion}

The three cases of AAK we presented in this series could be classified as severe, moderate, and mild, respectively, according to the criteria put forward by Lopez-Garcia et al. ${ }^{2}$ The corneal changes in AAK usually occur in patients in the first two decade. If left untreated, the peripheral corneal epitheliopathy advances to the pericentral and central cornea, leading to neovascularization, subepithelial fibrosis and stromal scarring. ${ }^{3}$

The present study showed that the in vivo morphology of central cornea varies based on the severity of the AAK. In the most severe case (case 1), the complete loss of normal corneal epithelium and the presence of goblet cells indicates the conjunctivalization of ocular surface. In contrast, the epithelial morphology was fairly normal in mildly affected eyes (case 3). The morphological changes in the corneal epithelium were consistent with previous studies. ${ }^{4,5}$ Our findings indicate that the in vivo morphology of corneal epithelium had a close correlation with the severity of AAK. LSCM might be a promising tool to classify the severity of AAK.

It is found that the subbasal nerve in AAK became sparse and thin, or even totally disappeared in the advanced stage. It is contradictory to Lagali's report, ${ }^{4}$ who demonstrated extremely dense subbasal nerve in aniridia cases. Our previous study shows that in patients 


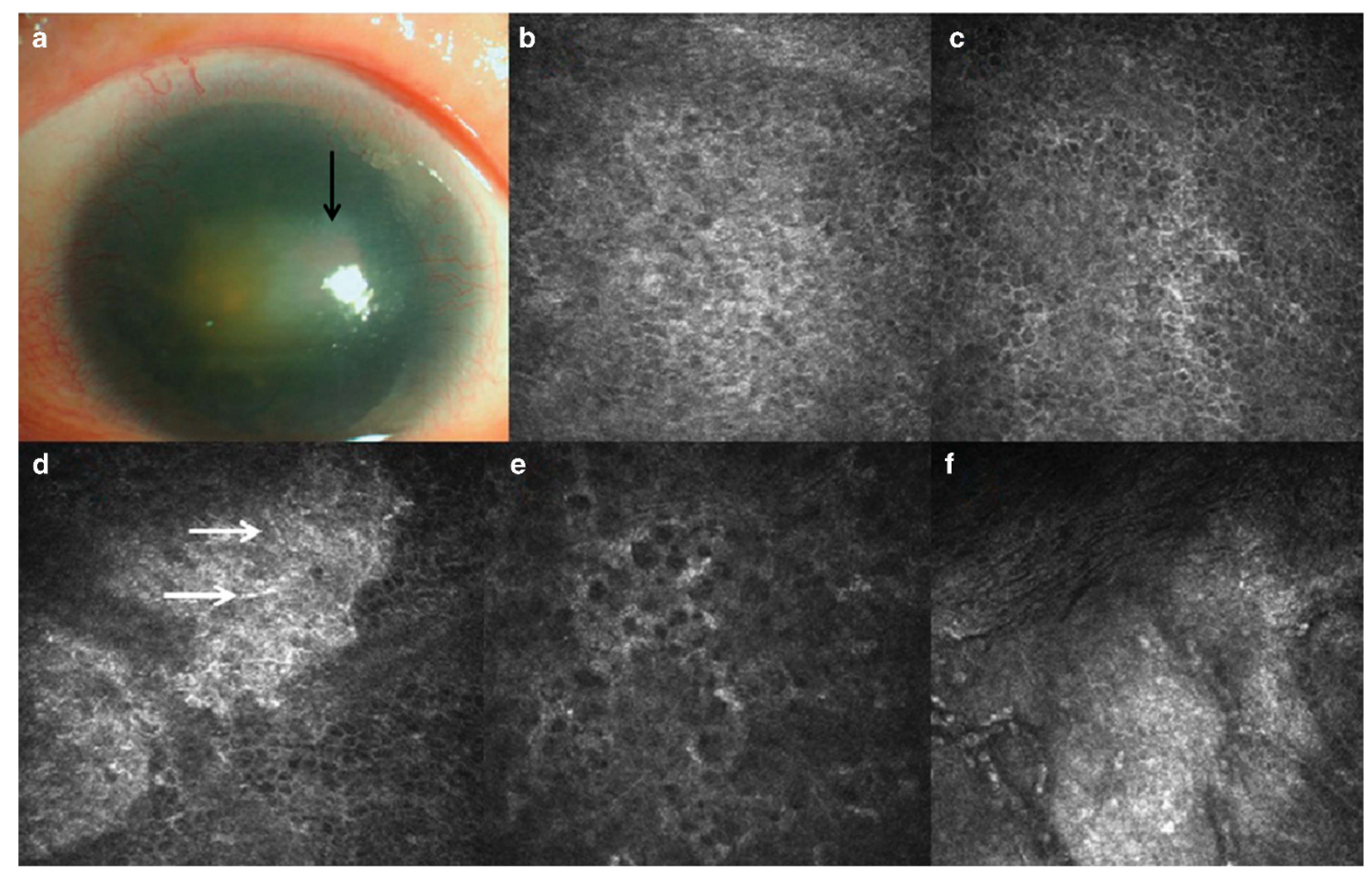

Figure 2 Clinical findings in case 2. (a) Slitlamp biomicroscopic photo shows focal subepithelial fibrosis at the central cornea (black arrow). Dense nuclear cataract was present in both the eyes. (b-e) Confocal microscopic images of the central cornea. The wing and basal epithelial cells were distinguishable (b, c). The subepithelial nerves were absent and dendritic cells (white arrows) were detected (d). Activated keratocytes were visible (e). (f) Confocal image of limbus shows the formation of fibrovascular tissues and the absence of Vogt palisades.

Table 1 The parameters of each layer and structure in cornea

\begin{tabular}{|c|c|c|c|c|c|c|c|c|c|c|c|c|c|c|}
\hline & \multicolumn{4}{|c|}{ Corneal epithelium (cells/mm²) } & \multicolumn{4}{|c|}{ Corneal stroma $\left(\right.$ cells $\left./ \mathrm{mm}^{2}\right)$} & \multicolumn{2}{|c|}{$\begin{array}{l}\text { Corneal endothelium } \\
\quad\left(\text { cells } / \mathrm{mm}^{2}\right)\end{array}$} & \multicolumn{2}{|c|}{$\begin{array}{l}\text { Corneal subbasal nerve } \\
\text { density }(\mu \mathrm{m} / \text { frame })\end{array}$} & \multicolumn{2}{|c|}{$\begin{array}{l}\text { Goblet cells in cornea } \\
\quad\left(\mathrm{cells} / \mathrm{mm}^{2}\right)\end{array}$} \\
\hline & \multicolumn{2}{|c|}{ Wing cells } & \multicolumn{2}{|c|}{ Basal cells } & \multicolumn{2}{|c|}{ Anterior stroma } & \multicolumn{2}{|c|}{ Posterior stroma } & \multirow[b]{2}{*}{$O D$} & \multirow[b]{2}{*}{ OS } & \multirow[b]{2}{*}{$O D$} & \multirow[b]{2}{*}{ OS } & \multirow[b]{2}{*}{$O D$} & \multirow[b]{2}{*}{ OS } \\
\hline & $O D$ & OS & $O D$ & OS & $O D$ & OS & $O D$ & OS & & & & & & \\
\hline Case 1 & NF & NF & NF & NF & ND & ND & ND & ND & ND & ND & ND & ND & $374 \pm 23$ & $365 \pm 35$ \\
\hline Case 2 & $3572 \pm 420$ & $2799 \pm 202$ & $4743 \pm 284$ & $3598 \pm 270$ & ND & $428 \pm 138$ & ND & $290 \pm 132$ & ND & ND & ND & ND & NF & NF \\
\hline Case 3 & $4784 \pm 294$ & $4748 \pm 429$ & $6427 \pm 526$ & $6585 \pm 496$ & $761 \pm 121$ & $829 \pm 145$ & $592 \pm 124$ & $630 \pm 135$ & $3050 \pm 213$ & $2813 \pm 268$ & $885.86 \pm 156.35$ & $957.22 \pm 148.77$ & NF & NF \\
\hline Healthy subject 1 & $4680 \pm 324$ & $4164 \pm 295$ & $6067 \pm 415$ & $6441 \pm 330$ & $867 \pm 105$ & $903 \pm 99$ & $680 \pm 135$ & $693 \pm 119$ & $2680 \pm 302$ & $2860 \pm 303$ & $946.94 \pm 104.16$ & $899.45 \pm 110.89$ & NF & NF \\
\hline
\end{tabular}

Abbreviations: ND, not distinguishable, NF, not found.

Healthy subject 1 is age- and gender-matched with case 1 and case 2 .

Healthy subject 2 is age- and gender-matched with case 3 .

with LSCD, the subbasal nerve density decreased, ${ }^{5}$ and we propose that the degeneration and fragmentation of Bowman's layer in aniridic cornea might be the etiology. The different severity of AAK in our study might account for the different findings. The alterations in the subbasal nerve plexus should be elucidated by further study with a larger population.

IVCM on the limbus reveals the various degree of destruction in Vogt palisades. It's notable that the degree of LSCD is more severe in case 1 , even though the patient was at the similar age in case 2 . The possible reason might be the cataract surgery that she underwent several months prior, in which scleral tunnel incision was used. It has been shown that AAK is often worsened after surgery that involves excessive manipulation of the limbus. ${ }^{6}$ These finding suggest that the surgical manipulation at the limbal area in aniridic patients should be less destructive.

In summary, IVCM would be a promising tool to identify LSCD and corneal epitheliopathy in aniridia 


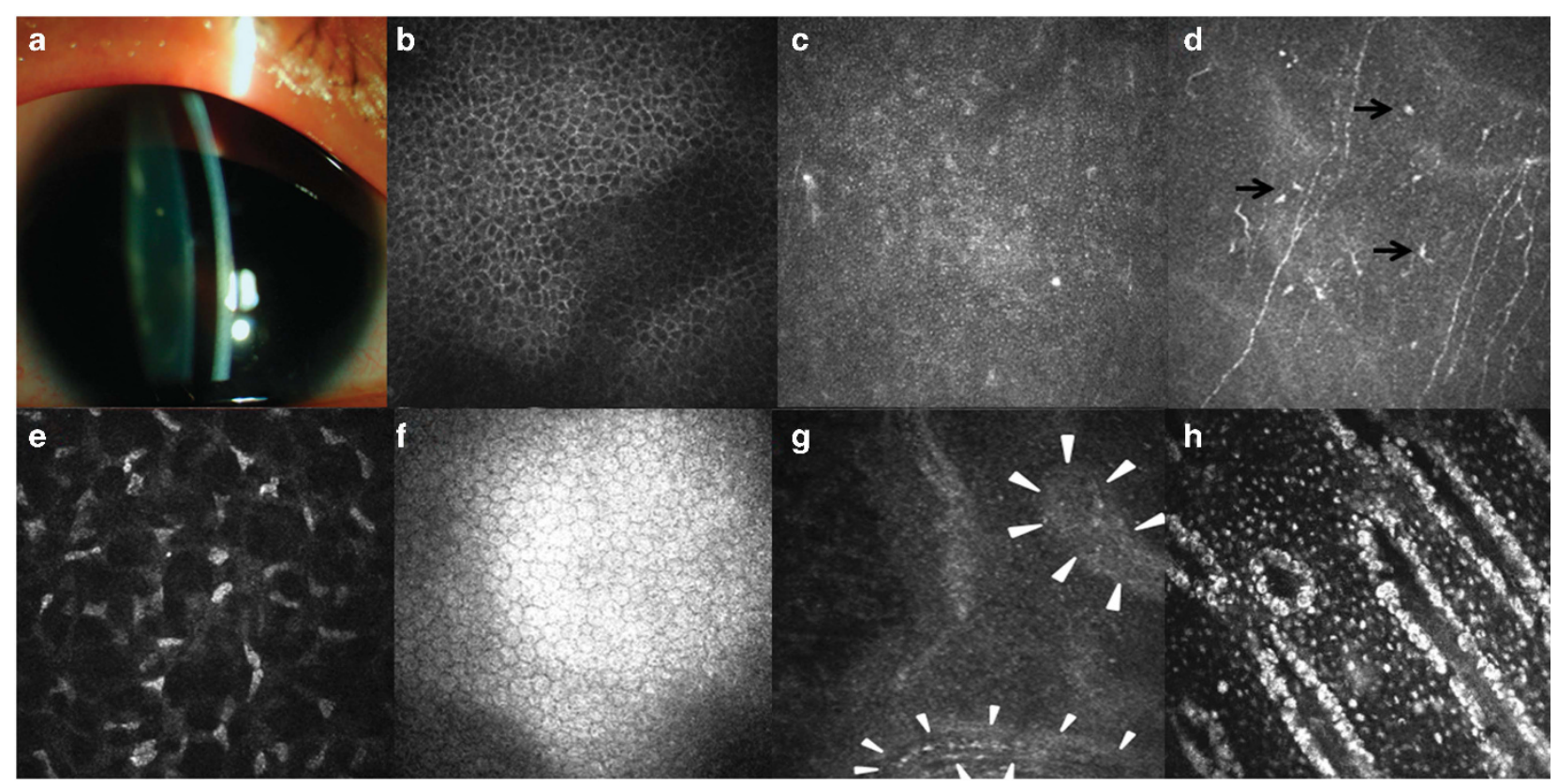

Figure 3 Clinical findings in case 3. (a) Slitlamp biomicroscopic photo shows a clear cornea. (b-f) Confocal microscopic images of the central cornea. The morphology of wing and basal epithelial cells was fairly normal $(b, c)$. The subbasal nerve plexus were visible, along with the infiltration of dendritic cells (black arrows) (d). The morphology of corneal stroma and endothelium was normal (e, $\mathrm{f}$ ). $(\mathrm{g}, \mathrm{h})$ Confocal images of the limbus. The palisade-like stromal cords were identified at limbus of this patient (white triangles) ( $\mathrm{g}$ ). Within the lower cord in the image, a slender blood vessel was visible. Panel (h) was the image taken from an age-matched healthy subject, revealing that hyperreflective cells were present around stromal cords in normal Vogt palisades.

with different severity. To quantify the morphological changes of AAK, further study with a larger amount of participants would be required.

\section{Summary}

What was known before

- Limbal stem cell deficiency (LSCD) is often involved in congenital aniridia.

- However, little is known regarding the in vivo morphological characteristics of the cornea and limbus in AAK.

What this study adds

- The morphological changes of cornea and limbus vary in AAK, and IVCM is a promising tool to determine the degree of LSCD in patients with AAK.

\section{Conflict of interest}

The authors declare no conflict of interest.

\section{Acknowledgements}

This work was supported by the National Natural Science Foundation of China $(81020108017,81270014)$.

\section{Disclaimer}

The authors have no proprietary or commercial interest in any of the materials discussed in this article.

\section{References}

1 Skeens HM, Brooks BP, Holland EJ. Congenital aniridia variant: minimally abnormal irides with severe limbal stem cell deficiency. Ophthalmology 2011; 118: 1260-1264.

2 López-García JS, García-Lozano I, Rivas L, MartínezGarchitorena J. Congenital aniridia keratopathy treatment. Arch Soc Esp Oftalmol 2006; 81: 435-444.

3 Whitson JT, Liang C, Godfrey DG, Petroll WM, Cavanagh HD, Patel D et al. Central corneal thickness in patients with congenital aniridia. Eye Contact Lens 2005; 31: 221-224.

4 Edén U, Fagerholm P, Danyali R, Lagali N. Pathologic epithelial and anterior corneal nerve morphology in early-stage congenital aniridic keratopathy. Ophthalmology 2012; 119(9): 1803-1810.

5 Deng SX, Sejpal KD, Tang Q, Aldave AJ, Lee OL, Yu F. Characterization of limbal stem cell deficiency by in vivo laser scanning confocal microscopy: a microstructural approach. Arch Ophthalmol 2012; 130: 440-445.

6 Lee H, Khan R, O'Keefe M. Aniridia: current pathology and management. Acta Ophthalmol 2008; 86: 708-715. 